\title{
Analysis of the Blocking Effect in Equivalence Classes Established by Match-to-Sample and Respondent-Type Training
}

\section{Kristopher Brown ${ }^{*}, 1$ \& Michael Clayton ${ }^{2}$}

${ }^{1}$ Department of Psychology, Youngstown State University, 1 University Plaza, Youngstown, OH 44555, USA

${ }^{2}$ Department of Psychology, Missouri State University, 901 S. National Ave. Springfield, MO 65897, USA

\begin{abstract}
Background/Objective: Blocking occurs when previous conditioning with one stimulus reduces, or blocks, conditioning to a second redundant stimulus added later in training or conditioning procedures. Previous research has suggested that blocking may occur during equivalence class formation. Although both match-to-sample and respondent-type training have been used to establish equivalence classes, blocking has only been studied using match to sample procedures. Previous research on blocking in equivalence class formation did not utilize control groups, limiting the conclusions drawn from those studies. Thus, the purpose of the present study was to compare match-to-sample and respondent-type training for their susceptibility to blocking in three-member equivalence classes using control conditions.
\end{abstract}

Method: A total of 27 participants were exposed to match-to-sample or respondent-type training procedures to form equivalence classes using a linear series training structure. Within each training procedure, one group of participants was exposed to a blocking condition and one was not.

Results: Results indicated that the blocking did not occur for the 4 of 6 participants who formed equivalence classes. Patterns of responding that would be expected if blocking occurred were seen in both experimental and control conditions.

Conclusions: Results obtained in the current study indicate that some processes other than blocking are involved when stimuli fail to enter equivalence classes. Future research should examine other processes involved when stimuli fail to enter into equivalence classes after training.

\section{Introduction}

Stimulus equivalence occurs when several untrained relations between stimuli emerge after direct training of only some relations between them [1]. For example, a student may be taught to select a picture of a $\operatorname{dog}(\mathrm{B})$ when an instructor presents the (A) auditory stimulus "dog" $(A \rightarrow B$ relation). Later, the student could be taught to select the printed word "DOG" $(C)$ when presented with the (B) picture of a dog $(B \rightarrow C$ relation). After this training is complete, a student who formed an equivalence class would be able to label the picture of a $\operatorname{dog}(B \rightarrow A)$, match the printed word "DOG" to the picture of a $\operatorname{dog}(C \rightarrow B)$, to identify the printed word "DOG" when given a spoken name $(A \rightarrow C)$, and read the printed word "DOG" $(C \rightarrow A)$ without direct instruction.

From a practical standpoint, the emergence of untrained relations is important because individuals can be taught skills and relations between stimuli without teaching each relation explicitly [2]. Equivalence has been established with different populations including adults, college students, children with neurodevelopmental disorders, typically developing children, and individuals with other psychological disorders such as anxiety and pathological gambling [3-5]. Theoretically, the emergence of untrained relations during equivalence class formation is important because it provides an objective model to study complex behavioral phenomena such as transfer of function, acquisition of symbolic reasoning, and even false memories [6-8].

\section{Training procedures in equivalence class formation}

Stimulus equivalence has traditionally been regarded as an operant phenomenon [9] in which match-to-sample (MTS) training is utilized to teach initial relations between stimuli that produce the emergent ones. In MTS training, an overt selection response is required from participants who are reinforced with feedback contingent on selecting the correct comparison stimulus that matches the sample during training sessions (i.e., selecting $B_{1}$ when presented with $A_{1}$ ).Tests for the emergence of untrained relations occur in the absence of reinforcement after these initial relations are taught. If the participant responds with a high level of accuracy on testing trials for emergent relations, it is inferred that the stimuli have joined in an equivalence class and now function interchangeably.

While the direct reinforcement used in MTS accounts for the acquisition of the explicitly taught relations, it does not account for the emergent relations that appear in testing phases $[9,10]$. This finding limits the extent to which operant conditioning alone can explain equivalence class formation because the emergent relations themselves occur without any explicit reinforcement or training. In an attempt to examine the necessary and sufficient conditions for the emergence of untrained relations, Leader and Barnes-Holmes [11] demonstrated a procedure that resembles respondent rather than operant conditioning procedures called respondent-type training $(\mathrm{ReT})$ that can reliably facilitate emergent relations between stimuli without any response requirement on the part of the participant. In ReT, the participant only needs to observe the stimuli as they occur in front of them. The stimuli are "paired" through contiguous temporal arrangement with one another. Shorter delays occur within pairs of *Corresponding Author: Kristopher Brown, Department of Psychology Youngstown State University, Youngstown, OH 44555, USA; E-mail: kjbrown@ysu.edu

Citation: Brown K, Clayton M (2020) Analysis of the Blocking Effect in Equivalence Classes Established by Match-to-Sample and Respondent-Type Training. Int J Psychol Behav Anal 6: 169. doi: https://doi.org/10.15344/2455-3867/2020/169

Copyright: (C) 2020 Brown. This is an open-access article distributed under the terms of the Creative Commons Attribution License, which permits unrestricted use, distribution, and reproduction in any medium, provided the original author and source are credited. 
stimuli and longer temporal delays occur between different pairs of stimuli [12]. For example, in training a three stimulus (A, B, and C) equivalence class with ReT, stimulus A would appear on the screen for 1 second. After this, the screen is cleared for half a second and stimulus $B$ would appear on the screen for 1 second $(A \rightarrow B)$. Lastly, a 3 second delay occurs in which the screen goes blank before a new trial begins with new stimuli to train a different relation in the same way (i.e., $B \rightarrow C$ ). While the training of relations between stimuli is completed using ReT training, an MTS procedure is still used to assess class formation [13].

Subsequent research has indicated that ReT reliably yields emergent relations in lieu of overt responses used in MTS training [12,14]. This procedure has several advantages compared to MTS, namely that participants do not have the opportunity to respond incorrectly in training due to position biases in the comparison field [15]. This procedure and its subsequent success in facilitating emergent relations has called into question what processes(s) may be involved in the formation of equivalence classes. Several studies have also been conducted to assess the relative effectiveness of MTS and ReT in facilitating the emergence of untrained relations and have not shown one method to be definitively more effective than the other $[7,16,17]$.

Complex Control in Equivalence Class Formation. In addition to different training procedures, the number of stimuli presented simultaneously in training has been manipulated to examine how this affects equivalence class formation. A complex sample consists of more than one element (i.e., A and B) and both elements can acquire stimulus control over responding [18]. For instance, instead of training $\mathrm{A} \rightarrow \mathrm{B} \rightarrow \mathrm{C}$, a complex stimulus consisting of $\mathrm{AB}$ can be conditionally related to $\mathrm{C}$ (i.e., $\mathrm{AB} \rightarrow \mathrm{C}$ ) where $\mathrm{A}, \mathrm{B}$, and $\mathrm{C}$ will still function independently on subsequent emergent relations tests (i.e., $C \rightarrow B$ and $\mathrm{C} \rightarrow \mathrm{A}$ ). Several researchers have examined complex stimulus control in equivalence class formation and found that the use of complex stimuli in training can be utilized to successfully facilitate equivalence class formation [19-21]. The use of complex stimuli in equivalence class formation is important because time may be saved in training if the members of the multi-element stimulus can exert control over responding on tests for emergent relations. Essentially, training with complex samples would reduce the number of relations that have to be initially taught but the number of emergent relationships would remain the same.

\section{The blocking effect}

Blocking occurs when previous conditioning with one stimulus reduces, or blocks, conditioning to a second stimulus when the stimuli are later presented as a compound [22]. In a traditional blocking preparation, the relationship between two stimuli are first taught (i.e., $\mathrm{A} \rightarrow \mathrm{B})$. Next, an additional stimulus $(\mathrm{X})$ is added to the A stimulus (i.e., $\mathrm{AX} \rightarrow \mathrm{B}$ ). Lastly, the relationships between all three stimuli are assessed. Blocking occurs if there is a reduced, or no response, to the $\mathrm{X}$ stimulus. This relation (i.e., $\mathrm{X} \rightarrow \mathrm{B}$ ) is "blocked" by the previous conditioning in the first phase $(A \rightarrow B)$.The $X$ stimulus essentially is "redundant" and provides no additional information when combined with the A stimulus which previously was paired with stimulus B. The blocking effect illustrates that temporal contiguity between stimuli is a necessary, but not sufficient condition for the development of stimulus control over responding [9]. In the example above, the $\mathrm{X}$ stimulus fails to develop a relationship with other stimuli even though it was presented concurrently with the A stimulus several times. Blocking has previously been demonstrated in animals such as rats, mollusks, and humans $[23,24]$. Blocking in equivalence classes would demonstrate a situation in which complex stimulus control would not develop over responding. That is, some otherwise expected relations between stimuli would be blocked by previous stimulus experience.

Rehfeldt, Dixon, Hayes, and Steele [25] were the first to examine how blocking could affect the inclusion of stimuli into equivalence classes later. Ten undergraduate students were trained to relate stimuli in a manner that resembled a blocking preparation. Students were first taught an $\mathrm{A} \rightarrow \mathrm{B}$ relationship until they reliably matched these stimuli to one another. A new stimulus (X) was then added with the A stimulus and presented simultaneously in additional trials $(A X \rightarrow B)$. Lastly, $\mathrm{B} \rightarrow \mathrm{C}$ was taught to the students. After this, tests for blocking and derived relations occurred. Evidence of blocking was evaluated by comparing scores on test trials for emergent relations when $\mathrm{X}$ was a sample or a comparison. If blocking occurred, scores on trials containing the $\mathrm{X}$ stimulus would be lower than other trials. Five of the 10 participants showed the formation of three 3 -member equivalence classes and subsequently showed evidence of a blocking effect. Participants who showed the formation of equivalence classes also had the lowest scores on tests for within-compound relations between the stimuli that comprised the compound stimulus (i.e., given $A_{1}$ select $\mathrm{X}_{1}$ and vice versa). In a similar experiment, Rehfeldt, Clayton, and Hayes [26] examined the blocking effect in equivalence classes with five-member equivalence classes. Six participants were trained to conditionally relate $A \rightarrow B, A \rightarrow C, A X \rightarrow B, A X \rightarrow C$, and $C \rightarrow D$. Evidence of blocking was evaluated by comparing scores on test trials for emergent relations when $\mathrm{X}$ was a sample or a comparison. The results demonstrated that only 2 participants displayed the formation of equivalence classes; one participant showed a reliable blocking effect while the other participant displayed the emergence of six-member classes, indicating that for one subject the stimulus that was expected to be blocked instead entered into the class with the other stimuli.

Results were mixed across the two studies that directly investigated blocking in equivalence class formation with some participants showing evidence of blocking and others not showing evidence of this effect. Both studies were limited in that they did not include a control group that did not undergo the blocking preparation. Additionally, no study on blocking in equivalence classes examined ReT training and if this training procedure would be susceptible to blocking. It is important to study blocking in ReT because blocking as a behavioral phenomenon was originally established in respondent (i.e., Pavlovian) conditioning experiments and is widely considered a respondent or Pavlovian phenomenon [27]. Thus, examinations of the susceptibility of the ReT procedure to blocking would provide a clearer understanding of the processes involved in equivalence class formation in a more general sense. Therefore, the purpose of the present study was to systematically replicate Rehfeldt, Dixon, Hayes, and Steele [25] and Rehfeldt, Clayton, and Hayes [26] and examine both MTS and ReT procedures to examine their relative susceptibility to the blocking effect with the addition of control conditions to gain better insight into the effects of blocking on equivalence class formation.

\section{Materials and Method}

\section{Participants, settings, and materials}

Participants were 27 undergraduate students enrolled at a state university in Northeast Ohio who were recruited using a sign-up sheet outside the psychology department. Informed consent was obtained 
Citation: Brown K, Clayton M (2020) Analysis of the Blocking Effect in Equivalence Classes Established by Match-to-Sample and Respondent-Type Training. Int J Psychol Behav Anal 6: 169. doi: https://doi.org/10.15344/2455-3867/2020/169

Page 3 of 8

from all participants before the study and approval was obtained from the universities' Institutional Review Board (IRB). Participants were offered extra credit for participating in the study. Extra credit was not contingent on their performance in the study. Each participant was assigned a unique subject number and was randomly assigned to one of the four groups: MTS control, MTS experimental, ReT control, or ReT experimental.

The training and testing sessions occurred in an $8.5 \times 4.5$ meter computer lab that contained 12 computers, tables, and chairs that was quiet and free from distractions. Each participant was tested individually. Participants sat at the table in front of the computer screen while the experimenter sat at a table behind them out of view. Each experimental session ranged from 30 minutes to 1 hour for each participant in the MTS condition depending on how quickly they acquired the initial relations. In ReT training, conditions ranged from 20 to 30 minutes and was shorter because no response was required from the participant in training.

Stimuli in all conditions consisted of twelve $5 \times 5 \mathrm{~cm}$ abstract symbols presented via computer using Microsoft PowerPoint ${ }^{\circ}$ software (Table 1). In MTS training; the participant touched the space bar to cycle through the slides presenting the stimuli. In ReT training, the stimuli were presented automatically with a timed presentation. Data were recorded using paper and pencil by the experimenter for all MTS training and testing phases. No data were recorded in ReT training sessions as no overt participant response was required in this condition.

\section{Experimental design, dependent variable, and interobserver agreement}

The relative susceptibility of MTS and ReT to blocking was evaluated by analyzing differences in percent correct on tests of emergent relationships that contained the redundant stimulus $\left(\mathrm{X}_{1-3}\right)$. During both MTS training and testing phases, a correct response was defined as selecting the appropriate comparison stimulus (i.e., pointing to or saying "left", "center", or "right") in the comparison field belonging to the same class as the sample (i.e., selecting $B_{1}$ when it was a comparison and $A_{1}$ was the sample and not selecting comparisons $\mathrm{B}_{2}$ or $\mathrm{B}_{3}$ ). An incorrect response in MTS training and testing trials was defined as the participant selecting any other stimulus in the comparison field. In all emergent relations testing phases, there was no feedback for responding. For this study, formation of a stimulus equivalence class was defined as scores of $77 \%$ or higher on all tests for emergent relations after initial training was complete.

An independent observer collected reliability data during 53\% of all MTS training sessions, $64 \%$ of all MTS testing sessions, and $41 \%$ of ReT testing sessions. The second observer did not provide any consequences or feedback to the participants at any time and was seated behind them so the recorded data could not influence participants. Percentage agreement was calculated using the total count method in which the smaller observed frequency of correct responses during inter observer agreement sessions is divided by the larger observed frequency of correct responses and multiplied by 100. Inter observer agreement was $100 \%$ in MTS training sessions, 99\% in MTS testing sessions (range: $95 \%-100 \%$ ), and $99 \%$ in ReT testing sessions (range: $97 \%$ to $100 \%$ ). Data were also collected on the integrity of the experimenter's response during MTS training (i.e., saying "That's correct" or "That's incorrect") on 53\% of MTS training trials and was calculated at $100 \%$.

\section{Procedure}

Respondent-Type Training: During ReT training, participants sat at the computer workstation which displayed on-screen instructions for the first phase of the experimental procedure. These instructions are adapted from Leader and Barnes-Holmes [11]:

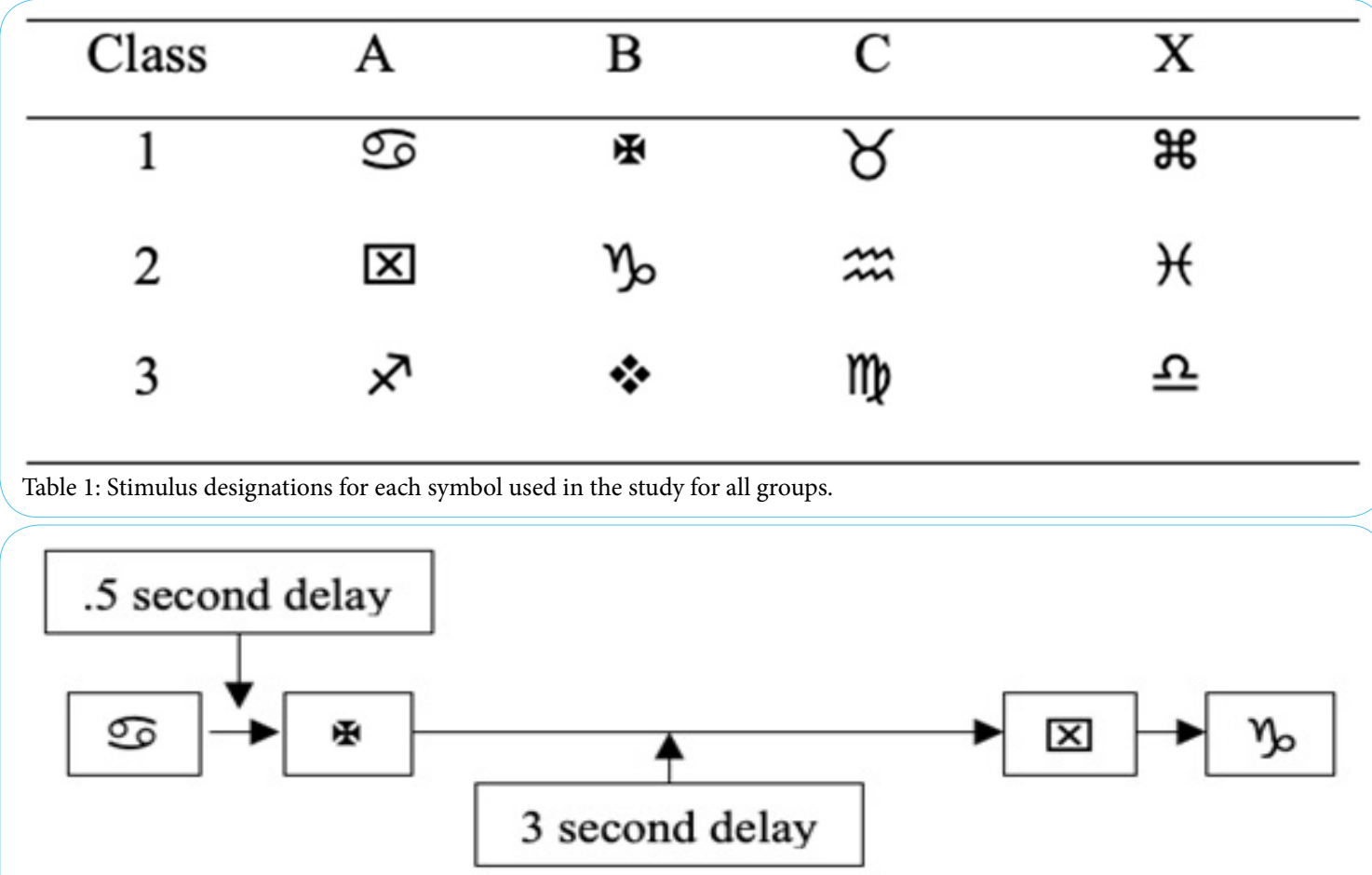

Figure 1: Schematic representation of respondent training sessionsfor $A \rightarrow B$ relations. 
"During the first stage of this experiment you will be presented with abstract shapes on the computer screen. Your job is to simply pay attention to the symbols as they appear. You should pay close attention to this first stage because it is relevant to the second stage of the experiment. Press the space-bar when you are ready to begin".

After this, ReT began and stimulus pairs were presented on the screen. Presentation consisted of the first stimulus or complex sample which was displayed for 1 second, followed by a .5 second within-pair delay. The second stimulus was presented for 1 second and was followed by a 3 second between-pair delay (Figure 1). This procedure was repeated in randomized loop until all individual pairs were presented 10 times for a total of 120 pairs in the experimental condition and 90 pairs in the control condition.

$A \rightarrow B$ respondent-type training. In this phase the A stimulus was presented, followed by the B stimulus in a randomized order, 10 times each, for 30 stimulus pairings. This phase only occurred in the ReT experimental group.

$A X \rightarrow B$ respondent-type training. In this phase a complex sample (AX) was initially presented followed by the $\mathrm{B}$ stimulus for each class $(A X \rightarrow B)$. Pairs were presented 10 times each for each class for a total of 30 pairs. Positions of the compound stimuli were randomly rotated between left and right but always appeared in the same location on the computer screen. This condition occurred in both the ReT experimental and control conditions.

$B \rightarrow C$ respondent-type training. In this phase the $B$ stimulus for each class was presented and then followed by the $C$ stimulus for each class. Each pair was presented 10 times for a total of 30 pairs. This condition occurred in both the ReT experimental and control conditions.

Mix $A X \rightarrow B$ and $B \rightarrow C$ respondent-type training. In this phase the $\mathrm{AX} \rightarrow \mathrm{B}$ and $\mathrm{B} \rightarrow \mathrm{C}$ relations were presented in a randomized order 15 times each for 30 trials. The positions of the stimuli in the compounds were randomly rotated between left and right by the experimenter but always appeared in the same location on the computer screen. This condition occurred in both the ReT experimental and control conditions.

Match-to-SampleTraining. During MTS training, participants were seated at the computer workstation which displayed on-screen instructions for the first phase of the experimental procedure. These instructions were as follows:

"During this stage of the experiment, your task is to find out which of the stimuli belong together. You will be presented with one symbol in the bottom of the screen and three more on the top of the screen. Your job is to select the one at the top that goes with the one on the bottom by telling the experimenter your choice by saying left, middle, or right to indicate the left, center, or right symbol as your choice. In some phases, you will receive feedback on your selection but in others you will not. The experimenter is always keeping your score, whether you get feedback or not. When you are ready to continue, please press space".

After this, MTS training began in the manner described above. To start an MTS trial, the sample stimulus appeared centered on the bottom portion of the screen and 3 comparison stimuli appeared on the top portion evenly spaced from left to right. All presentations of trials as well as the position of the comparison stimuli were randomized. Contingent upon a correct response in MTS training, the experimenter said "That's correct" before the next trial was presented. Incorrect responses resulted in the experimenter saying, "That's incorrect". Participants were required to score $86 \%$ correct on each of the MTS training phases to continue. If a participant failed to achieve a score of $86 \%$, the training began again. When a participant failed a phase four times, he/she was eliminated from the study. Thus, the number of minimum trials was 120 for the experimental and 90 for the control group, but the actual number of trials could be higher due to individual differences in responding during training phases.

$\boldsymbol{A} \rightarrow \boldsymbol{B}$ match-to-sample training. In this phase the sample stimulus on the bottom was an $A$ stimulus $\left(A_{1}, A_{2}\right.$, or $\left.A_{3}\right)$ and comparisons were all three of the $\mathrm{B}$ stimuli $\left(\mathrm{B}_{1}, \mathrm{~B}_{2}\right.$, and $\left.\mathrm{B}_{3}\right)$. The correct response in this condition was selecting the class consistent $\mathrm{B}$ comparison stimulus in the presence of the appropriate $A$ stimulus (i.e., $A_{1} \rightarrow B_{1}$ ). Each of these three relations was presented 10 times for a total of 30 trials. This phase occurred only in the MTS experimental group.

$A X \rightarrow B$ match-to-sample training. In this phase, a complex sample consisting of an A stimulus with the additional redundant stimulus was presented (i.e., $\left.\mathrm{AX}_{1-3}\right)$ and the $\mathrm{B}$ stimuli $\left(\mathrm{B}_{1-3}\right)$ were comparisons. The correct response was selecting the class consistent $\mathrm{B}$ stimulus in the presence of the correct complex sample (i.e., $\mathrm{AX}_{1} \rightarrow \mathrm{B}_{1}$ ). This phase occurred in both the MTS experimental and control conditions.

$B \rightarrow C$ match-to-sample training. In this phase the sample stimulus on the bottom was the $\mathrm{B}$ stimulus $\left(\mathrm{B}_{1-3}\right)$. Comparisons were all three of the $\mathrm{C}$ stimuli $\left(\mathrm{C}_{1-3}\right)$. The correct response was selecting the class consistent $C$ stimulus in the presence of the $B$ stimulus (i.e., $B_{1} \rightarrow C_{1}$ ). Each of these three relations was presented 10 times for a total of 30 trials. This phase occurred in both the MTS experimental and control conditions.

Mix $A X \rightarrow B$ and $B \rightarrow C$ match-to-sample training. This phase consisted of the presentation of 30 mixed trials of $A X \rightarrow B$ and $B \rightarrow C$ relations. The positions of the stimuli in the compound were randomly rotated between left and right, but always appeared in the same area of the screen. The correct response was to select the class consistent comparison described above (i.e., $\mathrm{AX}_{1} \rightarrow \mathrm{B}_{1}$ ). This phase occurred in both the MTS experimental and control conditions. Table 2 illustrates the relations taught and tested in this study.

\begin{tabular}{|l|l|l|}
\hline Condition & MTS & ReT \\
\hline Experimental & $\mathrm{A} \rightarrow \mathrm{B}, \mathrm{AX} \rightarrow \mathrm{B}, \mathrm{B} \rightarrow \mathrm{C}, \mathrm{Mix}$ & $\mathrm{A} \rightarrow \mathrm{B}, \mathrm{AX} \rightarrow \mathrm{B}, \mathrm{B} \rightarrow \mathrm{C}, \mathrm{Mix}$ \\
\hline Control & $\mathrm{AX} \rightarrow \mathrm{B}, \mathrm{B} \rightarrow \mathrm{C}, \mathrm{Mix}$ & $\mathrm{AX} \rightarrow \mathrm{B}, \mathrm{B} \rightarrow \mathrm{C}, \mathrm{Mix}$ \\
\hline
\end{tabular}

Table 2: Trained relations in both training procedures across conditions.

Tests for Emergent Relations and Blocking. After completion of all training relations, tests for emergent relations and blocking occurred for all participants in all groups. Before the testing phase, the following directions were displayed on the screen:

"In this stage you must look at the symbol at the bottom of the screen, and then choose one of the symbols or group of symbols at the top that it goes with. You are to tell the experimenter your choice by saying left, middle, or right to indicate the left, center, or right symbol as your choice. You will not be presented with any feedback for correct or incorrect answers in this portion of the experiment, but the experimenter is still recording your score. Press the space-bar to continue". 
Citation: Brown K, Clayton M (2020) Analysis of the Blocking Effect in Equivalence Classes Established by Match-to-Sample and Respondent-Type Training. Int J Psychol Behav Anal 6: 169. doi: https://doi.org/10.15344/2455-3867/2020/169

Page 5 of 8

After this, each participant was presented with the same procedure described in MTS training but with for emergent relations for all classes of stimuli three times, resulting in a total of 63 testing trials (Figure 2). No feedback for responding was presented in this phase of the experiment. After participants finished tests for emergent relations their participation concluded and they were debriefed, offered the opportunity to ask questions of the experimenter, and were dismissed.

\section{Results and Discussion}

Of the 15 participants who began in one of the MTS conditions, 14 completed the initial conditional discrimination training before moving on to emergent relations and blocking tests. In total, 4 of the 14 participants (28\%) who completed MTS training demonstrated the formation of equivalence classes: 2 in the experimental condition and 2 in the control condition. Table 3 shows the number of trials participants who formed equivalence classes took to complete conditional discrimination training for each phase in the MTS condition. In the ReT training condition, 2 out of 12 participants showed the emergence of equivalence classes, both of whom were in the ReT experimental condition. No participants in the ReT control condition displayed emergence of full equivalence classes.

The presence of blocking was evaluated by examining the differences between test trials that contained the redundant stimulus (X) which was programmed to be blocked and test trials that did not contain the $\mathrm{X}$ stimulus for the 6 participants who showed the formation of equivalence classes. In the MTS experimental condition, Participant 103 displayed the formation of three 3-member equivalence classes and scores consistent with the blocking effect ( $M=91.67$ on non-X trials compared to $M=25.92$ on trials containing the X stimulus). Conversely, Participant 102 underwent the same preparation, but scores were not consistent with a blocking effect $(M=97.22$ on non-X trials compared to $M=92.59$ on trials containing the X stimulus). Instead, Participant 102 displayed the formation of three 4-member equivalence classes that included the X stimulus. In the MTS control group which did not contain the blocking preparation, Participant 204 displayed the emergence of three 3-member equivalence classes and displayed responses that would be typical of the blocking effect ( $M=94.45$ on non-X trials compared to $M=59.26$ on trials containing the $\mathrm{X}$ stimulus) even though no such preparation occurred in this condition. Participant 201 (who underwent the same preparation) did not display this effect $(M=91.67$ on non-X trials compared to $M=$ 96.30 on trials containing the $\mathrm{X}$ stimulus), with scores indicating the emergence of three 4-member equivalence classes including the $\mathrm{X}$ stimulus (Figure 3).

In the ReT condition, two participants showed the emergence of full equivalence classes, both of whom were in the ReT experimental group that contained the programmed blocking preparation. Both participants displayed no evidence of blocking despite exposed to conditions that were expected to result in blocking. Participant 302 demonstrated scores indicating the emergence of four 3-member equivalence classes $(M=97.22$ on non-X trials compared to $M=$ 92.59 on trials containing the X stimulus). Similarly, Participant 305 displayed the emergence of three 4-member equivalence classes by scoring perfectly on all tests $(M=100.00$ on non-X trials and $M=$ 100.00 on trials containing the $\mathrm{X}$ stimulus). No participants showed the emergence of all three 3-member equivalence classes in the ReT control group. In sum, both the participants who displayed the emergence of equivalence classes in ReT did not display evidence of the blocking effect despite undergoing a preparation designed to program this effect (Figure 4).

\section{Conclusions}

The current study addresses past research limitations on blocking in equivalence class formation by utilizing control conditions while subsequently expanding the literature by investigating blocking in ReT trained equivalence classes. On the surface, results in the MTS conditions were consistent with Rehfeldt, Clayton, and Hayes [26] in that some participants showed evidence of a strong blocking effect

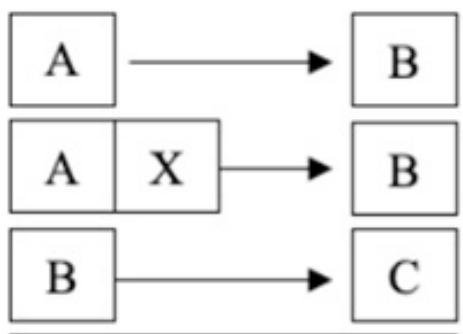

Mix AX-B, B-C

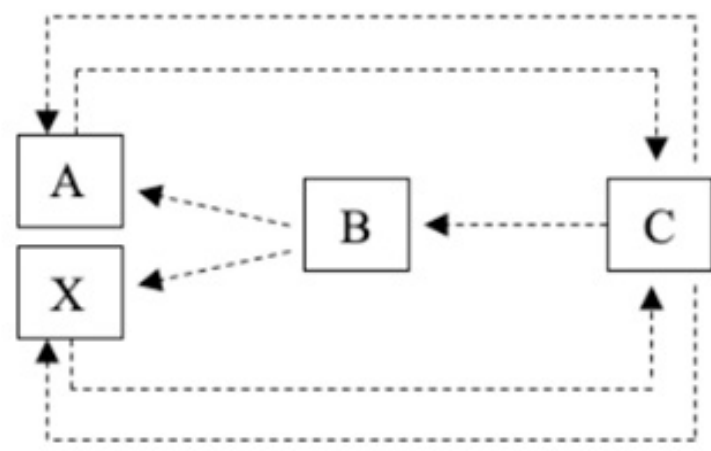

Figure 2: Trained (left) and emergent (right) relations in the current study using the linear training structure. Trained relations are in solid lines while derived relations tested for are in dotted lines.

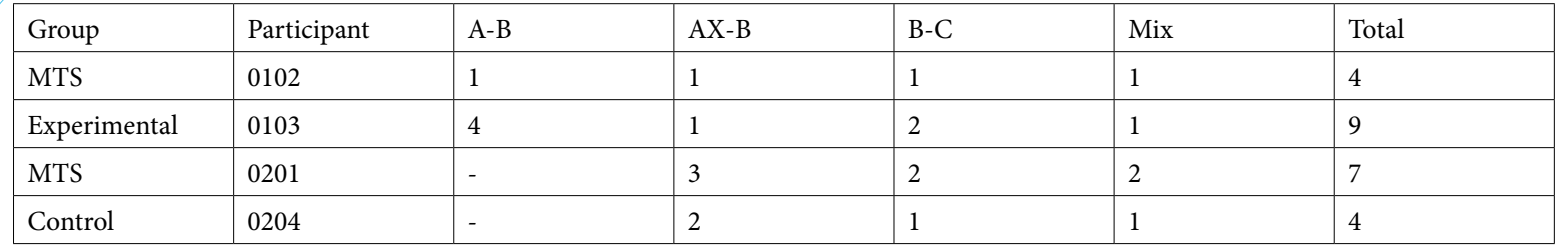

Table 3: Trials to criterion during training for MTS groups. 
Citation: Brown K, Clayton M (2020) Analysis of the Blocking Effect in Equivalence Classes Established by Match-to-Sample and Respondent-Type Training. Int J Psychol Behav Anal 6: 169. doi: https://doi.org/10.15344/2455-3867/2020/169

while the other did not. However, the ability to compare the presence of blocking between experimental and control conditions proved to be important in shedding additional light on the blocking phenomena in equivalence class formation. One participant in each of the MTS control and MTS experimental condition scored in a manner that was inconsistent with expectations based on their experimental preparation. Results in the ReT condition were also inconsistent at best with the demonstration of blocking despite programming for such an effect. Two participants trained with ReT training showed the emergence of full equivalence classes in the ReT experimental condition. Rather than demonstrating a blocking effect, these two participants subsequently displayed the inclusion of the redundant stimulus into equivalence classes despite being in the experimental group which contained the blocking preparation. Of note, Participant 305 scored perfectly on all tests of emergent relations including those on which the redundant stimulus was included.

The results of this study do not support the blocking effect as a singular explanation for the inclusion or exclusion of stimuli into equivalence classes. Across both MTS and ReT experimental conditions, the majority ( 3 out of 4 ) participants failed to demonstrate a blocking effect and instead showed inclusion of the redundant stimulus into 4-member stimulus equivalence classes despite undergoing the blocking preparation. In the MTS control condition, one participant also displayed scores indicating a blocking effect despite not undergoing an experimental preparation to program for
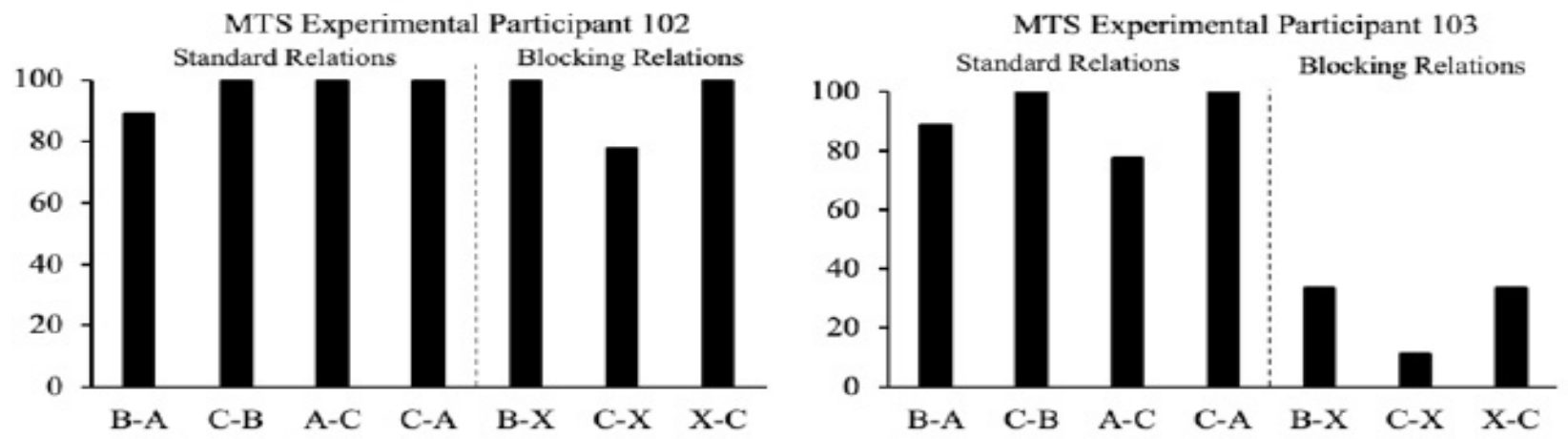

MTS Control Participant 201

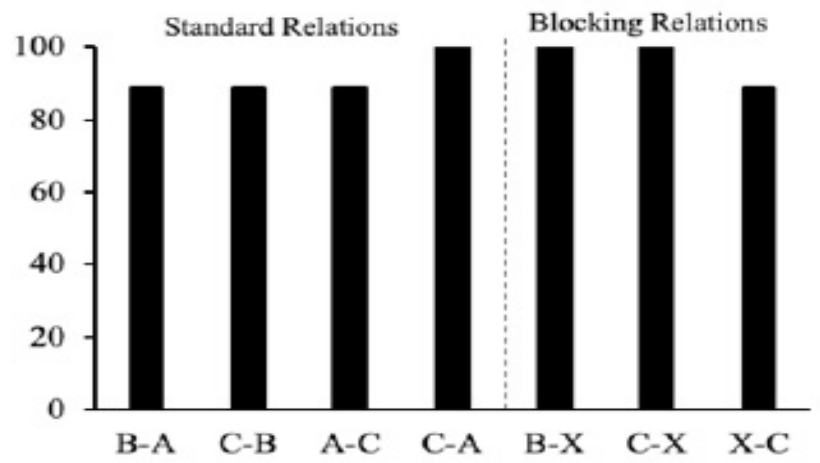

MTS Control Participant 204

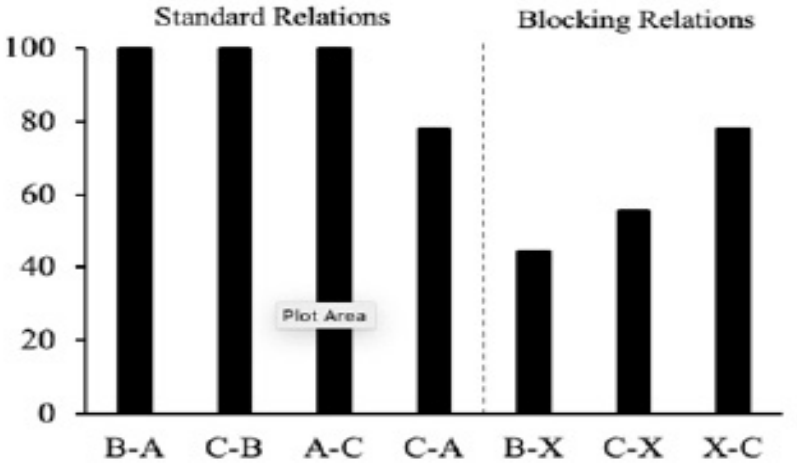

Figure 3: Scores on tests for emergent relations for standard trials and trials containing the redundant stimulus including in the blocking preparation for participants in the MTS experimental (top) and MTS control conditions (bottom).
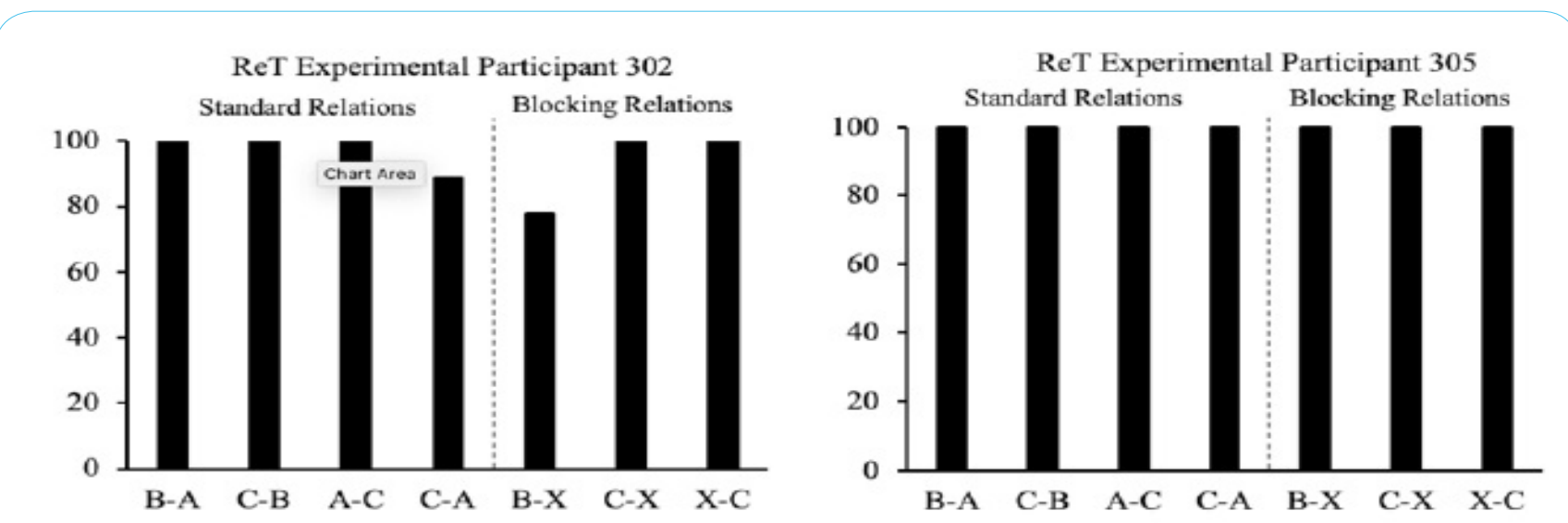

Figure 4: Scores on tests for emergent relations for standard trials and trials containing the redundant stimulus including in the blocking preparation for participants in the ReT experimental condition. 
this effect. Although no participants in the ReT control condition formed classes, the fact that those in the ReT experimental condition showed the redundant stimulus in equivalence classes indicates that blocking did not occur. These results are also consistent with recent failures to replicate the blocking effect in traditional preparations with humans that have been reported in the literature [28].

Future research should examine the other processes involved in determining the inclusion/exclusion of stimuli into stimulus equivalence classes beyond blocking alone. Individual differences in attending to the stimuli could provide a simple explanation for differences in scores. Differential attending to redundant stimuli involved in blocking experiments has been documented in the literature [29]. In the MTS control condition, it could have been the case that participants simply attended to A stimulus rather than the $\mathrm{X}$ stimulus when they were presented in a complex sample. This responding pattern would still have resulted in correct responses in MTS training despite not attending to the $\mathrm{X}$ stimulus. However, the failure to attend to both stimuli would have been demonstrated in low scores on tests with the individual X stimulus. The opposite could have been the case in the MTS experimental condition. It is possible this individual simply attended to the $\mathrm{X}$ stimulus that was added after initial $\mathrm{A} \rightarrow \mathrm{B}$ training in the $\mathrm{AX} \rightarrow \mathrm{B}$ condition even though it essentially did not add any additional information. An interesting extension of the current research would be to require an observing response on the part of the participants during initial ReT training [30]. Requiring participants to engage in such a response during training could function to increase attention to the task and subsequently affect scores on emergent relation tests.

The ReT training utilized in this study is not the only type in the literature on training emergent relations. In the current study, the ReT preparation was similar to trace conditioning procedures in that the two stimuli to be related were presented after short delays and never appeared together [31]. A similar, but not identical procedure to the ReT procedure used in the behavioral literature is called the stimulus pairing observation procedure and has received empirical support for facilitating emergent relations [32-34]. This procedure is more similar to delay conditioning in which the two stimuli are presented simultaneously rather than after a temporal delay [35]. Future research should examine if different preparations based on respondent procedures are differentially affect equivalence class formation.

Another point for future research was noted anecdotally during experimental sessions. As discussed, both participants in the ReT condition who formed all three classes also showed the inclusion of the redundant stimulus and the subsequent formation of four 3-member equivalence classes. In post-experimental debriefings, both participants who displayed the emergence of full equivalence classes in ReT indicated that they formulated names for each of the stimuli that appeared on the screen. Although anecdotal, these indications may provide some support of Horne and Lowe's [36] naming hypothesis, which identifies naming as the basic unit of verbal behavior and suggests that naming stimuli in initial training may facilitate equivalence class formation [37]. Previous research has demonstrated that providing verbally labeled response options (i.e., equivalent versus non-equivalent) to participant during initial training has resulted in higher class formation than those who were not provided such options [38]. Future research could examine this effect by requiring participants in some conditions to formulate labels for the abstract stimuli before progressing through ReT training of equivalence classes and compare scores to individuals who are not made to apply such labels to the stimuli. This information may help researchers gain a more complete understanding of the sufficient and necessary conditions for equivalence class formation trained with MTS and ReT procedures.

\section{Limitations}

Two limitations of the current study should be addressed in future research. First, the current study utilized a linear series $(A \rightarrow B \rightarrow C)$ training model, which typically produces lower scores on emergent relation trials than other training procedures $[39,40]$. Only 6 out of 27 participants (22\%) overall between both conditions displayed emergence of all classes relations and formations of three 3-member equivalence classes. For comparison, 7 out of 16 participants (44\%) in the original stimulus equivalence studies on blocking displayed the emergence of equivalence classes. Future research into blocking may benefit from the use of training procedures that have been demonstrated to facilitate emergent relations more reliably such as one to many (OTM) and many to one (MTO) training preparations, both of which are more effective than linear series training [41].

Secondly, in keeping consistent with the previous research on blocking in class formation the current study utilized a mixed training condition following $\mathrm{B} \rightarrow \mathrm{C}$ training in all four groups. This phase may have served to weaken or dilute the blocking effect in the two experimental groups. This training was more recent and included direct reinforcement on $\mathrm{AX} \rightarrow \mathrm{B}$ relations in the MTS experimental condition and may have weakened the control exerted by the $\mathrm{A}$ stimulus alone. This would have made it more likely that AX would function as a compound rather than $\mathrm{A}$ on its own (i.e., AX instead of A). Typical animal research on blocking does not contain similar phases after initial phases occur. Future research should examine the effects of eliminating this extra phase to examine if evidence of blocking may become more consistent as a result.

While the current study was basic, it has applied implications. As mentioned, stimulus equivalence has been applied as a technology in educational and clinical settings with individuals with neurodevelopmental disabilities and other psychological conditions. Previous research has demonstrated that equivalence classes can readily be formed utilizing complex samples. Blocking and other variables that affect class formation would present a situation in which equivalence procedures would fail to produce otherwise expected emergent relations. The data in this study from two participants showed that the ReT training procedure yielded lower equivalence class formation rates than MTS, but when they did emerge, all stimuli were included in 3 four-member equivalence classes. Future research should examine the relationship between different types of equivalence class training and their susceptibility to conditions that could disrupt class formation. It may be the case that classes formed with different training procedures are more resistant to the influence of other variables. This information could contribute to developing instructional procedures that yield emergent relations that are more robust and beneficial to the learner.

\section{Competing Interests}

The author declare that there is no competing interests regarding the publication of this article. 
Citation: Brown K, Clayton M (2020) Analysis of the Blocking Effect in Equivalence Classes Established by Match-to-Sample and Respondent-Type Training. Int J Psychol Behav Anal 6: 169. doi: https://doi.org/10.15344/2455-3867/2020/169

Page 8 of 8

\section{References}

1. O'Neill J, Rehfeldt RA, Ninness C, Muñoz BE, Mellor J, et al. (2015) Learning Skinner's verbal operants: Comparing an online stimulus equivalence procedure to an assigned reading. Anal Verbal Behav 31: 255-266.

2. Critchfield TS (2018) Efficiency is everything: Promoting efficient practice by harnessing derived stimulus relations. Behav Anal Prac 11: 206-210.

3. Dymond S, Bennett M, Boyle S, Roche B, Schlund M, et al. (2017) Related to anxiety: Arbitrarily applicable relational responding and experimental psychopathology research on fear and avoidance. Perspect Behav Sci 41: 189-213.

4. Rehfeldt RA (2011) Toward a technology of derived stimulus relations: An analysis of articles published in the Journal of Applied Behavior Analysis, 1992-2009. J Appl Behav Anal 44: 109-119.

5. Walker BD, Rehfeldt RA (2012) An evaluation of the stimulus equivalence paradigm to teach single-subject design to distance education students via blackboard. J Appl Behav Anal 45: 329-344.

6. Ruiz-Sánchez LJ, Luciano C, Guinther PM (2019) Derived false memories using a respondent-type (ReT) procedure. J Exp AnalBehav 111: 12-27.

7. Clayton MC, Hayes LJ (2004) A comparison of match-to-sample and respondent-type training of equivalence classes. Psychol Rec 54: 579-602.

8. Dixon MR, Belisle J, Stanley CR, Daar JH, Williams LA, et al. (2016) Derived equivalence relations of geometry skills in students with autism: An application of the PEAK-E Curriculum. Anal Verbal Behav 32: 38-45.

9. Rehfelt RA, Hayes $\amalg$ (1998) The operant-respondent distinction revisited Toward an understanding of stimulus equivalence. Psychol Rec 48: 187-210.

10. Solares L, Fryling MJ (2018) Further evaluation of the stimulus pairing observation procedure with children with autism spectrum disorder. Anal Verbal Behav 35: 85-93.

11. Leader G, Barnes-Holmes D (1996) Establishing equivalence relations using a respondent-type training procedure. Psychol Rec 46: 685-706.

12. Avellaneda $M$, Menéndez J, Santillán $M$, Sánchez F, Idesis $S$, et al. (2016) Equivalence class formation is influenced by stimulus contingency. Psychol Rec 66: 477-487.

13. Dickins DW (2015) A simpler route to stimulus equivalence? A replication and further exploration of a "simple discrimination training procedure" (Canovas, Debert and Pilgrim 2014). Psychol Rec 65: 637-647.

14. Ruiz SL, Luciano C, Guinther PM (2019) Derived false memories using a respondent-type (ReT) procedure. J Exp Anal Behav 111: 12-27.

15. Omori M, Yamamoto J (2013) Stimulus pairing training for Kanji reading skills in students with developmental disabilities. Res Dev Disabil 34: 1109 1118.

16. Kinloch JM, McEwan J, Foster TM (2013) Matching-to-sample and stimuluspairing-observation-procedures in stimulus equivalence: The effect of number of trials and stimulus arrangement. Psychol Rec 63: 157-174.

17. Leader G, Barnes-Holmes D (2001) Matching-to-sample and respondenttype training as methods for producing equivalence relations: Isolating the critical variable. Psychol Rec 51: 429-444.

18. Stromer R, McIlvane WJ, Serna RW (1993) Complex stimulus control and equivalence. Psychol Rec 43: 585-598.

19. Carpentier F, Smeets PM, Barnes-Holmes D (2000) Matching compound samples with unitary comparisons: Derived stimulus relations in adults and children. Psychol Rec 50: 671-685.

20. Groskreutz NC, Karsina A, Miguel CF, Groskreutz MP (2010) Using complex auditory-visual sample to produce emergent relations in children with autism. J Appl Behav Anal 43: 131-136.

21. Rosales R, Maderitz C, Garcia YA (2014) Comparison of simple and complex auditory-visual conditional discrimination training. J Appl Behav Anal 47: 437-442.

22. Luque D, Vadillo MA, Gutiérrez-Cobo MJ, Le Pelley ME (2016) The blocking effect in associative learning involves learned biases in rapid attentional capture. Q J of Exp Psychol 71: 522-544.

23. Prados J, Alvarez B, Acebes F, Loy I, Sansa J, et al. (2013) Blocking in rats, humans, and snails using a within-subjects design. Behav Processes 100 23-31.

24. Stock AK, Gohil K, Beste C (2017) Blocking effects in non-conditioned goaldirected behaviour. Brain Struct Funct 222: 2807-2818.
25. Rehfeldt RA, Dixon MR, Hayes LJ, Steele A (1998) Stimulus equivalence and the blocking effect. Psychol Rec 48: 647-664.

26. Rehfeldt RA, Clayton M, Hayes LJ (1998) Blocking the formation of 5 member equivalence classes using complex samples. Mex J Behav Anal 24 279-292.

27. Vandbakk M, Olaff HS, Holth P (2020) Blocking of stimulus control and conditioned reinforcement. Psychol Rec 1: 1-14.

28. Maes E, Krypotos AM, Boddez Y, Alfei Palloni JM, D'Hooge R, et al. (2018) Failures to replicate blocking are surprising and informative-Reply to Soto (2018). J Exp Psychol General 147: 603-610.

29. Beesley T, Le Pelley ME (2011) The influence of blocking on overt attention and associability in human learning. J Exp Psychol Anim Behav Process 37: 114-120.

30. Soares-Filho PSD, de Carvalho LM, de Moraes Hamasaki EI, Campos HC, Perez WF, et al. (2019) Manual-observing procedure: An alternative to the investigation of stimulus control and equivalence classes in matching-tosample. Psychol Rec 69: 165-174.

31. Connor DA, Gould TJ (2016) The role of working memory and declarative memory in trace conditioning. Neurobiol of Learn Mem 134: 193-209.

32. Byrne BL, Rehfeldt RA, Aguirre AA (2014) Evaluating the effectiveness of the stimulus pairing observation procedure and multiple exemplar instruction on tact and listener responses in children with autism. Anal Verbal Behav 30: $160-169$

33. Rosales R, Rehfeldt RA, Huffman N (2012) Examining the utility of the stimulus pairing observation procedure with preschool children learning a second language. J Appl Behav Anal 45: 173-177.

34. Smyth S, Barnes-Holmes D, Forsyth JP (2006) A derived transfer of simple discrimination and self-reported arousal functions in spider fearful and non-spider-fearful participants. J Exp Anal Behav 85: 223-246.

35. Halverson HE, Khilkevich A, Mauk MD (2018) Cerebellar processing common to delay and trace eyelid conditioning. J Neurosci 38: 7221-7236.

36. Horne PJ, Lowe CF (1996) On the origins of naming and other symbolic behavior. J Exp Anal Behav 65: 185-241.

37. Carp CL, Petursdottir AI (2015) Intraverbal naming and equivalence class formation in children. J Exp Anal Behav 104: 223-240.

38. Tovar ÁE, Torres-Chavez A, Ruiz A (2015) Effects of verbal-labeled responses on stimulus class formation in a compound stimulus procedure. Mex Behav Anal 41: 68-85.

39. Arntzen E (2012) Training and testing parameters in formation of stimulus equivalence: Methodological issues. Europ J Behav Anal 13: 123-135.

40. Saunders RR, Green G (1999) A discrimination analysis of training structure effects on stimulus equivalence outcomes. J Exp AnalBehav 72: 117137.

41. Sadeghi P, Arntzen E (2018) Eye-movements, training structures, and stimulus equivalence class formation. Psychol Rec 68: 461-476.

Int J Psychol Behav Anal

ISSN: 2456-3501

IJPBA, an open access journal

Volume 6. 2020. 169 